\title{
Article \\ Characterizing THz Scattering Loss in Nano-Scale SOI Waveguides Exhibiting Stochastic Surface Roughness with Exponential Autocorrelation
}

\author{
Brian Guiana ${ }^{\dagger}$ and Ata Zadehgol $*,+$ (D) \\ Department of Electrical and Computer Engineering, University of Idaho, Moscow, ID 83844-1023, USA; \\ bguiana@uidaho.edu \\ * Correspondence: azadehgol@uidaho.edu; Tel.: +1-208-885-9000 \\ † These authors contributed equally to this work.
}

check for

updates

Citation: Guiana, B.; Zadehgol, A.

Characterizing THz Scattering Loss in Nano-Scale SOI Waveguides Exhibiting Stochastic Surface Roughness with Exponential Autocorrelation. Electronics 2022, 11, 307. https://doi.org/10.3390/ electronics11030307

Academic Editors: Giulio Antonini,

Daniele Romano and Luigi Lombardi

Received: 24 November 2021

Accepted: 16 January 2022

Published: 19 January 2022

Publisher's Note: MDPI stays neutral with regard to jurisdictional claims in published maps and institutional affiliations.

Copyright: (c) 2022 by the authors. Licensee MDPI, Basel, Switzerland. This article is an open access article distributed under the terms and conditions of the Creative Commons Attribution (CC BY) license (https:// creativecommons.org/licenses/by/ $4.0 /)$.

\begin{abstract}
Electromagnetic (EM) scattering may be a significant source of degradation in signal and power integrity of high-contrast silicon-on-insulator (SOI) nano-scale interconnects, such as optoelectronic or optical interconnects operating at $100 \mathrm{~s}$ of $\mathrm{THz}$ where two-dimensional (2D) analytical models of dielectric slab waveguides are often used to approximate scattering loss. In this work, a formulation is presented to relate the scattering (propagation) loss to the scattering parameters (S-parameters) for the smooth waveguide; the results are correlated with results from the finitedifference time-domain (FDTD) method in 2D space. We propose a normalization factor to the previous 2D analytical formulation for the stochastic scattering loss based on physical parameters of waveguides exhibiting random surface roughness under the exponential autocorrelation function (ACF), and validate the results by comparing against numerical experiments via the 2D FDTD method, through simulation of hundreds of rough waveguides; additionally, results are compared to other 2D analytical and previous 3D experimental results. The FDTD environment is described and validated by comparing results of the smooth waveguide against analytical solutions for wave impedance, propagation constant, and S-parameters. Results show that the FDTD model is in agreement with the analytical solution for the smooth waveguide and is a reasonable approximation of the stochastic scattering loss for the rough waveguide.
\end{abstract}

Keywords: dielectric slab waveguide; discrete filtering; exponential autocorrelation; FDTD; optical interconnects; photonics; random roughness; stochastic scattering loss; scattering parameters; S-parameters

\section{Introduction}

Nano-scale SOI optical interconnects, comprised of silicon/silicon-dioxide $(\mathrm{Si} / \mathrm{SiO})_{2}$ dielectric waveguides operating at 100 s of terahertz $(\mathrm{THz})$, constitute an increasingly important building block of modern integrated circuits, where the high-tech market demands smaller form-factors and wavelengths. Considering the non-ideal manufacturing process, random imperfections in the surfaces of nano-scale dielectric waveguides may cause significant signal degradation and power attenuation, as EM waves propagate through the interconnect structure, where the loss is primarily due to EM wave scattering with surface roughness of the waveguide [1-5]. Therefore, the characterization of scattering loss is a topic of significant interest to the scientific community [1-10].

The three-dimensional (3D) structure of SOI optical interconnects poses certain challenges to its analytical and numerical modeling; thus, the stochastic scattering loss observed in nano-scale $\mathrm{THz}$ SOI interconnects is often approximated using 2D planar models of the dielectric slab waveguide exhibiting surface roughness. The $2 \mathrm{D}$ analogue is useful for analytically $[5,8,10]$ characterizing the effect of scattering loss on the power attenuation 
of light waves, and is used as a comparison for both experimental analysis (of physical waveguides) [1-4] and numerical analysis $[1,2,6,7,9]$.

In 1983, Kuznetsov and Haus [11] published their work on using the 3D volume current method (VCM) to evaluate the radiation loss in dielectric waveguide structures. Their work includes analysis of single-line, two-line coupled, and three-line coupled waveguide structures, in the absence of random surface roughness. In 1990, Lacey and Payne [5] released their seminal work analyzing 2D planar waveguides exhibiting random surface roughness for a single-line waveguide structure. Their work applies Green's functions to the structure, operating in the transverse-electric-to-z $\left(\mathrm{TE}^{Z}\right)$ mode, as an approximation for scattering loss in 3D optical interconnects, and later in 1994 [8] it was updated to use normalized waveguide parameters. In 2005, Barwicz and Haus [6] expanded on both of those developments by applying the 3D VCM to single-line waveguides exhibiting random surface roughness. In each of these cases, despite having relatively simple geometries, the solutions are formulated around complicated integral equations, and the solutions only become more complicated as the geometry becomes more complex, for example by adding roughness, multiple tightly-coupled lines, arbitrary-shaped lines or grating, etc., thus limiting the application of integral-based solutions. An effective workaround to the integral-equation complication is to reformulate the problem around differential-equations, leading to the FDTD method. A version of the FDTD method based on wavelets is used in [2], but the details of the FDTD formulation are not included. The FDTD method is also used in [1] through the software tool Lumerical, but again details of the FDTD methodology are absent.

The major contributions of the present work are as follows. (1) We provide the FDTD methodology for analysis of 2D dielectric waveguides exhibiting random surface roughness, operating in the $\mathrm{TE}^{\mathrm{z}}$ mode. (2) We propose a methodology for the extraction of S-parameters, and we apply that methodology to the characterization of scattering loss. (3) We improve the computational efficiency of this model by using filtering techniques to attenuate numerical noise from simulation results, thereby allowing for the use of a relatively coarse spatial and temporal discretization while retaining the integrity of numerical results. (4) While the integral-based VCM has increasing complexity as the geometry becomes more complex, the FDTD method is especially well-suited for arbitrary waveguide geometries and arbitrary surface roughness profiles. To keep this presentation simple and concise, we chose to apply the methodology to a single line; however, it can easily be adapted to multiple tightly-coupled lines. (5) We provide the Python [12] code titled Optical Interconnect Designer Tool (OIDT) [13] which features multi-CPU-core support for parallelized FDTD, as an open-source software package [14] hosted on GitHub through a public repository under the GNU GPL v3.0 license [15], to encourage further exploration and (inter)national collaboration on optical interconnect research.

While the FDTD method implemented via a serial programming paradigm would be computationally expensive, its highly parallelizable nature may provide a potential path to a computationally expedient solution; thus, herein we begin to explore this potential by developing a parallelized implementation of FDTD with a traditional Yee-based algorithm [16,17] and convolution perfectly matched layer (CPML) [18] boundaries, to characterize the scattering loss in dielectric slab waveguides exhibiting surface roughness.

The remainder of this paper is organized as follows. Section 2.1 outlines the physical waveguide structure analyzed throughout this paper. Section 2.2 establishes the details of the FDTD model being used, where Section 2.2.1 provides additional details on the discretization and application of random roughness profiles to the FDTD environment. Section 2.2.2 details the filtering technique used to improve numerical measurements from FDTD simulations. Section 2.2.3 addresses the coordinate transformations used to move between the analytical solution and the FDTD model. In Section 2.3, we provide the methodology for S-parameter extraction in 2D FDTD. In Section 2.3.2, we formulate the relations between the S-parameter matrix and the scattering loss $\alpha$. In Section 2.4, we propose an updated equation for computing the stochastic scattering loss $\alpha$ based on 
physically realistic waveguide parameters and define its components, including a discussion on the exponential ACF. In Section 2.5, we validate the FDTD model by correlating against analytical expressions for the wave impedance, the propagation constant, and the ideal S-parameter matrix. In Section 3, we discuss the numerical results of FDTD, and the potential sources of error between FDTD and the analytical model. In Section 3.1, we compare our results against those of other investigators. In Section 3.2, we examine the dependence of $\alpha$ on input power and formulate the mode normalization $N_{F}$. We conclude with closing remarks in Section 4.

\section{Methods}

\subsection{The Waveguide Structure}

Optical interconnects are comprised of spatially 3D waveguide structures with a certain surface roughness profile which may not vary much relative to the smooth (flat) waveguide's width. Often, 2D models of dielectric slab waveguides with the same height and similar material parameters are used to analyze the 3D waveguide. This allows the analysis to be decomposed into two modes: (1) transverse electric (TE) and (2) transverse magnetic (TM).

Here, we analyze the structure in Figure 1 for the TE mode. We start by defining the coordinate grid in the $\hat{x}-\hat{z}$ plane, and orient the device to operate with infinite extent in both the $\hat{y}$ and the $\hat{z}$ directions, where the waveguide length and power flow are along $\hat{z}$ and the waveguide height is infinite along $\hat{y}$.

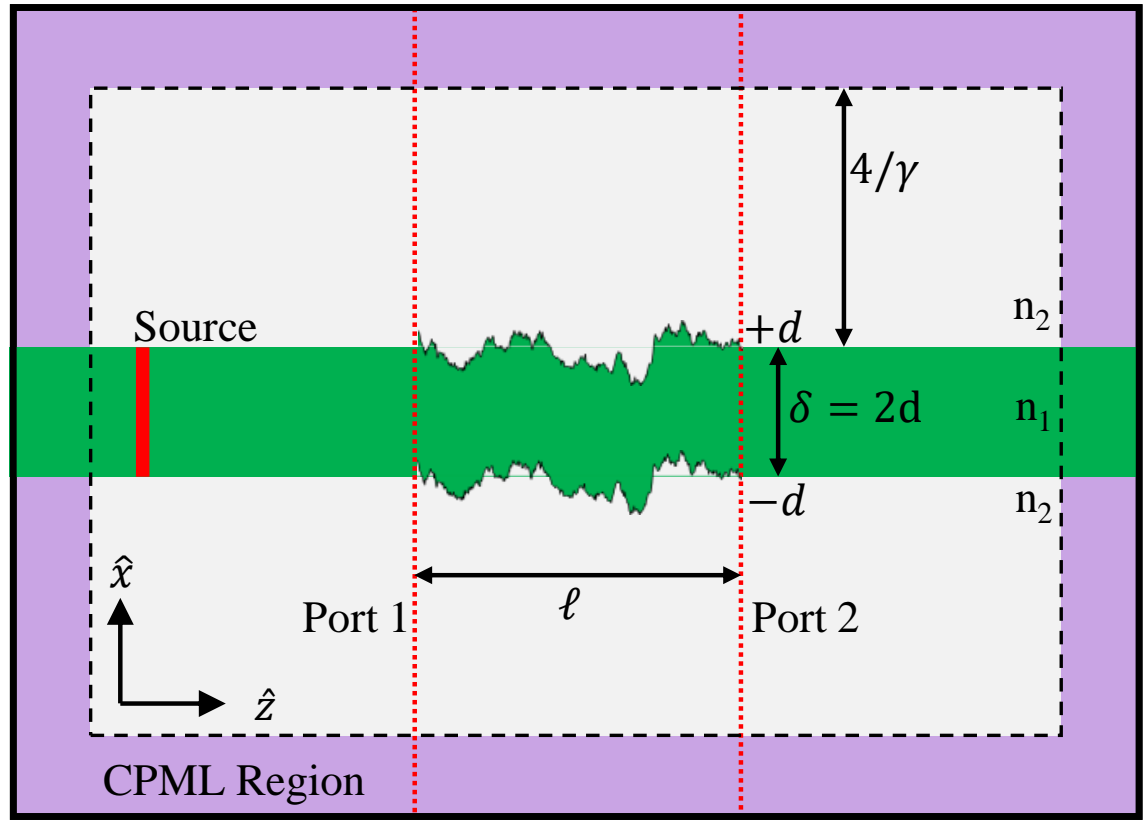

Figure 1. The baseline dielectric slab waveguide structure.

The dielectric slab waveguide consists of two regions, the core and the cladding. The core has a refractive index of $n_{1}$, and the cladding has a refractive index of $n_{2}$, where $n_{1}>n_{2}$. The core region has a finite nominal width, which is typically denoted as two half-widths. Here, the width is $\delta=2 d$, where $d$ is the half-width used throughout this work. The fields in the waveguide are assumed to be time harmonic (with $e^{j \omega t}$ dependence, where $\omega(\mathrm{rad} / \mathrm{s})$ is the angular frequency) in nature with the E-field taking the form in (1).

$$
\mathbf{E}(x, z)=\hat{y} \Phi(x) e^{-(\alpha+\beta)) z},
$$

where $\beta=n_{\mathrm{eff}} k_{0}(\mathrm{rad} / \mathrm{m}), n_{\mathrm{eff}}$ is the effective index found via the effective index method (EIM) [10], $k_{0}$ is the free-space wave number, $\alpha$ is the attenuation constant resulting 
from sidewall roughness, and $\Phi(x)$ is a piece-wise function with its components defined in (2)-(4). In (2) the term $A_{\mathcal{e}}$ is a scaling constant.

$$
\begin{gathered}
\Phi(x)= \begin{cases}A_{e} \cos (\kappa x) & |x| \leq d \\
A_{e} \cos (\kappa d) e^{-\gamma(|x|-d)} & |x|>d\end{cases} \\
\kappa^{2}=n_{1}^{2} k_{0}^{2}-\beta^{2} \\
\gamma^{2}=\beta^{2}-n_{2}^{2} k_{0}^{2}
\end{gathered}
$$

Random perturbations exist along the boundary between the core and cladding, resulting in the surface roughness profile. We use an exponential ACF to describe the surface roughness by its standard deviation $\sigma$ and its correlation length $L_{\mathcal{c}}$. The mean of the profile is 0 , so the random perturbations are not included in the nominal width. We further assume the cladding extends infinitely outward from the core region. The FDTD environment used in this paper is described fully in $[7,14,19]$, and in Section 2.2.

\subsection{The FDTD Environment}

The nominal waveguide structure may expediently fit into 2D FDTD analysis. We start by converting the nominal structure into uniform discrete cells with side length $\Delta x$. The temporal resolution is then set at the Courant stability limit [20] for 2D FDTD, where the background material is set to the cladding medium. We apply the CPML [18] to the exterior of the computational domain, thereby simulating infinite space with minimal reflections and computational cost.

We define a length $\ell$ over which we generate and discretize a random profile; the remaining FDTD cells create an extra buffer space to allow for modal waves to settle, after leaving the source point and before reaching the recording (numerical measurement) point. Each source and recording location are designated by a separate port, e.g., a single optical line would be characterized by two ports, with one port at each end of the line.

Once the roughness profile is ready, it is applied as the core and cladding boundary between ports. Referring to Figure 1, we place a source condition along $\hat{x}$ in a vertical line of cells across the entire opening of the waveguide, where the distance between the source and CPML is more than 10 cells, while we may approximate infinite space with the CPML, we still need to retain a buffer space in the cladding between the waveguide and the CPML boundary. To capture the intricacies of the interactions of the EM fields in both the core and cladding regions, we need to set the cladding size appropriately. Therefore, it is necessary to capture as much of the E-field as possible. Note, in (2) the E-field magnitude decays exponentially in the cladding region with a rate of $1 / \gamma$, and we can use that behavior to set the cladding buffer size. At a distance of $4 / \gamma$ from the core/cladding boundary, the E-field magnitude at the edge of the simulation space is no more than $2 \%$ of the E-field magnitude at the core/cladding interface, and it only decays further from there; thus, the cladding region size is set accordingly, as in Figure 1.

Data are collected in the form of E-field values at ports 1 and 2 along the first line of cells adjacent to the rough region. These points are recorded at each time-step for the duration of the FDTD analysis. In post-processing, we take the recorded time-domain E-field values and convert them to the frequency domain with the fast Fourier transform (FFT) [21]. We then numerically integrate the E-field over the recorded line of cells, resulting in a frequency dependent voltage with which further analysis may be performed.

We set up the FDTD grid based on the waveguide geometry, material parameters, and desired frequency range. The geometry is set up as shown in Figure 1 , where $n_{1}=3.5$ and $n_{2}=1.5$. We additionally set the fundamental frequency as $f_{0}=194.8 \mathrm{THz}$ (corresponding to source wavelength $\lambda_{s}=1.54 \mu \mathrm{m}$ ). Using the core refractive index, we find the minimum phase velocity $v_{\min }(\mathrm{m} / \mathrm{s})$. Using the fundamental frequency, we assign our desired maximum frequency as $f_{\max }=N_{H} f_{0}(\mathrm{~Hz})$, where $N_{H}$ is the number of desired harmonics above 
the fundamental. Using both the minimum phase velocity and the maximum frequency, we find the minimum wavelength simulated in the FDTD scheme with $\lambda_{\min }=v_{\min } / f_{\max }$. Then, our spatial discretization is $\Delta x=\lambda_{\min } / 30$. At $N_{H}=2, \Delta x \approx 7.3 \mathrm{~nm} /$ cell. We set the time-step $\Delta t$ at the Courant limit based on the cladding material which has the largest possible phase velocity in the FDTD environment, such that $\Delta t=\frac{\Delta x}{\sqrt{2} v_{\text {clad }}}$.

The total grid is 5554 cells $\times 247$ cells $(\hat{z} \times \hat{x})$ with 26,997 time-steps. We use 40 layers of CPML as the absorbing boundary condition because in our experience 30-40 CPML layers provides good correlation of wave impedance within $1 \%$. Along $\hat{x}$, the core region is centered and the nominal full width measures 36 cells, where the remaining 300 cells are evenly distributed on either side of the core as cladding. Along $\hat{z}, \ell=4092$ cells, and the remaining 1858 cells are evenly distributed to each port region.

The computations are done by using a workstation with two Intel ${ }^{\circledR}$ Xeon ${ }^{\circledR}$ E5-2687W v3 CPUs (40 logical cores), operating at $3.10 \mathrm{GHz}$. Each simulation occupies less than $410 \mathrm{MB}$ of RAM and is completed in roughly $300 \mathrm{~s}$ (or $5 \mathrm{~min}$ ).

\subsubsection{Verifying the Validity of Discretized Roughness Profiles}

We start by assigning a target for $\sigma_{\mathrm{o}}, L_{\mathcal{C}_{0}}$, and $\mu_{0}$, where $\mu$ designates the mean in this subsection. These parameters are then normalized by the spatial discretization step-size $\Delta x$ value used in the FDTD simulation to yield $\left\{\sigma=\sigma_{0} / \Delta x, L_{c}=L_{\mathcal{c}_{o}} / \Delta x, \mu=0\right\}$. The discrete values are passed into the Pyspeckle [22] Python library which uses the methods in [23] to generate random profiles; this generation process returns an array of a specified size with floating point values quantifying the surface perturbation. As was the case in [7], a linear offset is added to the aforementioned floating point array to ensure that all values are positive. The offset array is then cast to integer values via the floor function and the same linear offset is subtracted from the now integer array, where the final discrete array has parameters $\sigma^{\prime}, L_{c}^{\prime}$, and $\mu^{\prime}$. The error between input $(\sigma)$ and output $\left(\sigma^{\prime}\right)$ parameters may be quite large, due to the discretization process. However, we may circumvent this issue by constraint-based generation of profiles, described below.

We set a percentage tolerance for the normalized input parameters $\left\{\sigma, L_{c}, \mu\right\}$ and we check that the output parameters $\left\{\sigma^{\prime}, L_{c^{\prime}}^{\prime} \mu^{\prime}\right\}$ fit the input parameters within the prescribed tolerance. If a profile does not meet the criteria it is discarded and a new profile is generated. In our numerical experiments, the tolerance is specified by $\sigma^{\prime} \in[0.9 \sigma, 1.1 \sigma]$ and $L_{c}^{\prime} \in$ $\left[0.9 L_{c}, 1.1 L_{c}\right]$, and $\mu^{\prime} \in[-0.01,+0.01]$.

We find $\sigma^{\prime}$ and $\mu^{\prime}$ via built-in Numpy functions std and mean, respectively. We may estimate the $L_{c}^{\prime}$ value that fits the autocorrelation data, as explained next. We start by finding the autocorrelation of the generated discretized surface profile using the Pyspeckle autocorrelate function which provides a normalized array with its maximum value occurring at $\zeta=0$. Note the autocorrelation of the generated profile tracks an exponential ACF up to the correlation length, as can be seen in Figure 2. With that in mind, we apply a root finding technique to determine $L_{c}^{\prime}$ while using $R_{X X}\left(L_{c}^{\prime}\right)=e^{-1}$ as the reference value. We then subtract $e^{-1}$ from the discrete ACF and find the root closest to $\zeta=0$, which is the correlation length of the discrete ACF. We may then compare the $L_{c}^{\prime}$ with $L_{\mathcal{C}}$ to determine the validity of the generated discretized profile.

For samples with parameter values $\Delta x=11 \mathrm{~nm}$ and $\sigma=9 \mathrm{~nm}$, and knowing that the probability distribution function (PDF) of the random process is normal in nature, we know that $99 \%$ of values in the final array will be contained in the range $\pm 3 \sigma \approx \pm 2.46$. Applying the floor function to this range results in the discrete set $\{-3,-2,-1,0,1,2\}$ which may cause significant differences between output values $\left\{\sigma^{\prime}, L_{c}^{\prime}, \mu^{\prime}\right\}$ and input values $\left\{\sigma, L_{c}, \mu\right\}$. 


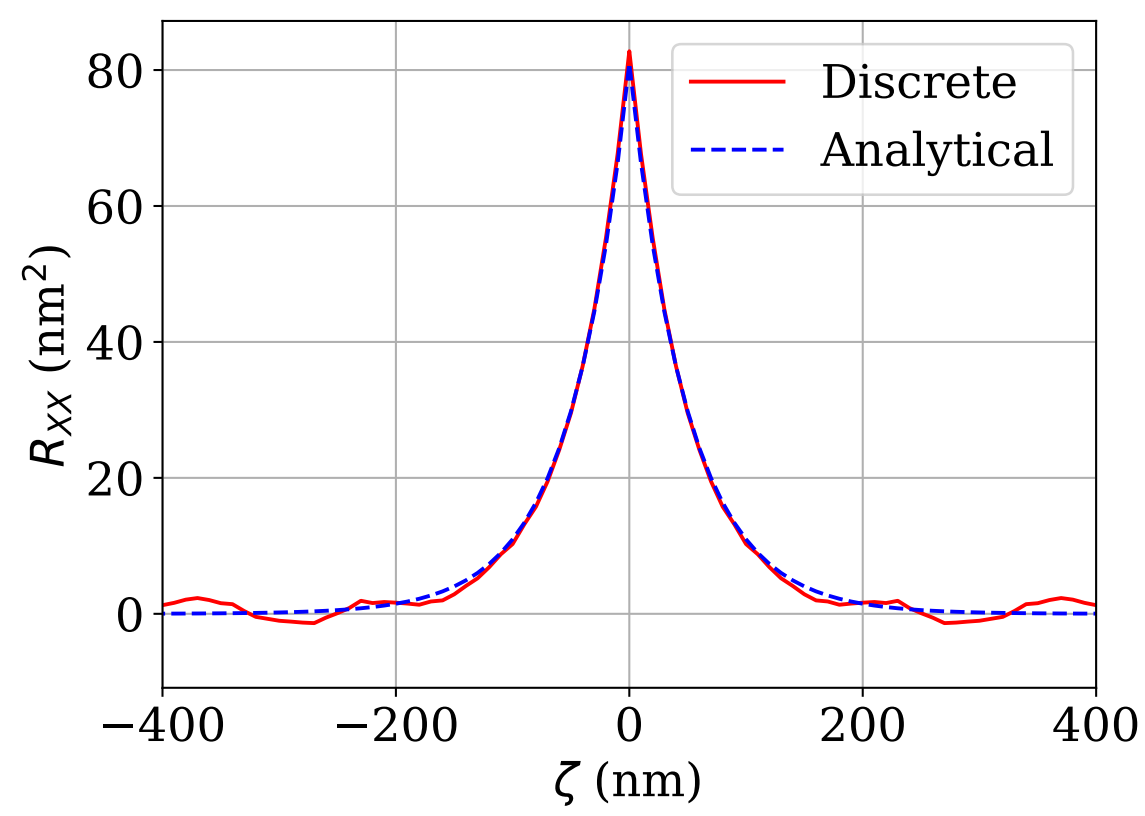

Figure 2. Example ACF with input parameters $\sigma=9 \mathrm{~nm}$ and $L_{c}=50 \mathrm{~nm}$. The discrete trace is generated with 5000 samples.

Figure 2 compares a discretized exponential ACF comprised of 5000 samples against the continuous analytical (18). Even at this small sample size, the discretized profile still correlates well to the ideal ACF up to $\zeta=L_{c}$, but after that point, there is noticeable noise. At $\zeta=0 \mathrm{~nm}$ the discretized and continuous analytical ACFs do not line up perfectly. The misalignment at $\zeta=0 \mathrm{~nm}$ may be remedied by normalizing the analytical ACF to match the discretized ACF, using $\sigma^{\prime}$ instead of $\sigma$.

\subsubsection{FDTD Noise Reduction}

Numerical experiments involving waveguides with random surface roughness result in a certain amount of noise in $\alpha$. We may observe that in the initial calculation, labeled unfiltered, there is rapid oscillation around the trend; such oscillations are undesirable and cause scattering loss readings to vary between numerical experiments. This issue may be resolved by the use of a moving-average function over the frequency range of interest; this technique is often used to reduce noise levels in digital signals [24]. We use (5) to reduce the noise level at each frequency point, where $N$ is the number of samples to either side the reference index $k$.

$$
\alpha_{\text {Filtered }}[k]=\frac{1}{2 N+1} \sum_{p=-N}^{N} \alpha_{\text {Unfiltered }}[k+p]
$$

The rolling average function is effective at reducing noise levels, but due to the smoothing effect it also introduces its own set of numerical distortions. However, the introduced error is small when $N$ is small. As such, we use $N=5$ to generate the filtered curve, where the rapid oscillations have been reduced but the trend remains mostly unchanged.

\subsubsection{Modal Transformations and Coordinate Mapping}

The geometry used for the characterization of scattering loss from random surface perturbations, shown in Figure 1, is based on the geometry from Figure 5 in [10]. The fields in [10] are described as $\mathrm{TE}^{\mathrm{Z}}$, since $E_{z}=0$ and $\frac{\partial}{\partial_{y}}=\partial_{y}=0$, and by using (56), (59), and (60) in [10] we know the non-zero field components are $\left\{E_{y}, H_{x}, H_{z}\right\}$, while $E_{z}=E_{x}=H_{y}=0$. The $\mathrm{TE}^{z}$ field configuration may also be obtained from (6-72) in [25] and setting $\partial_{y}=0$, and it is mathematically identical as two other modes; specifically, setting $\partial_{y}=0$ in (6-64) in [25] yields the $\mathrm{TM}^{\mathrm{y}}$, and in (6-74) in [25] yields $\mathrm{TE}^{\mathrm{x}}$. 
In our geometry of Figure 1, there exists a single nonzero E-field component along the invariant (infinite) direction $(\hat{y})$, and two nonzero H-field components along the finite directions $(\hat{z}, \hat{x})$ which may be interpreted as either height or width [10]. Out of the three mathematically equivalent modes $\left(\mathrm{TE}^{\mathrm{z}}, \mathrm{TM}^{\mathrm{y}}, \mathrm{TE}^{\mathrm{x}}\right)$, we choose the $\mathrm{TE}^{\mathrm{z}}$ field configuration here, as it aligns best with the physical interpretation of the physical waveguide with propagation along $\hat{z}$ (length), a transverse E-field along $\hat{y}$ (width or height), and H-field components along $\hat{x}$ and $\hat{z}$.

Our FDTD simulations are based on the traditional Yee algorithm in a 2D lattice, as formulated in ([17], ch. 3). Our FDTD formulation is derived with the assumption that $H_{z}=0$ and $\partial_{z}=0$, resulting in the $\mathrm{TM}^{\mathrm{z}}$ mode with field components $\left\{E_{z}, H_{x}, H_{y}\right\}$. This FDTD lattice may initially appear to be in conflict with our analytic formulation; however, note that the E-field has a single nonzero component along the infinite (invariant) direction, and the H-field has two nonzero components. Since we can assign the FDTD geometry in an arbitrary manner, we choose to orient $H_{x}$ along the length and $H_{y}$ along the width (or height), resulting in a field configuration with the same orientation as the analytical formulation but with a rotated coordinate grid. We can rotate the coordinate grid of the analytical field configuration such that it results in a configuration identical to the FDTD fields by the steps shown in Figure 3.

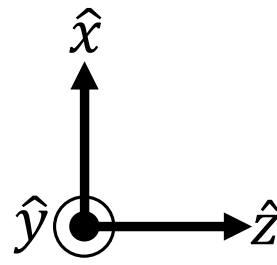

(a)

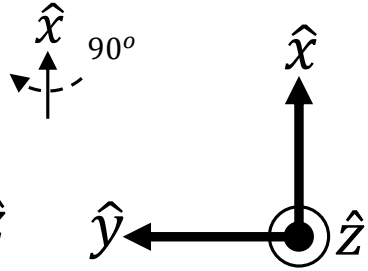

(b)
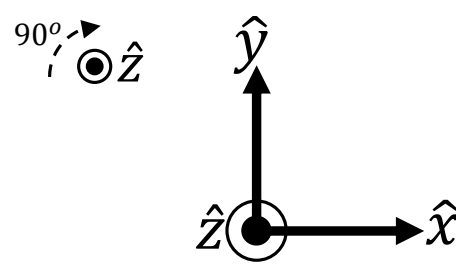

(c)

Figure 3. Coordinate grid rotation steps. (a) Initial orientation of analytical formulation for $2 \mathrm{D} \mathrm{TE}^{\mathrm{Z}}$. (b) Intermediary rotated mapping. (c) Final mapped orientation of analytical formulation is identical to the FDTD formulation for 2D TM ${ }^{\mathrm{z}}$.

In Figure 3, starting with the analytical expression in (a), we rotate the coordinate grid twice. The first rotation is $90^{\circ}$ around $\hat{x}$ from $\hat{z}$ toward $\hat{y}$; this produces the grid in (b). The second rotation is $90^{\circ}$ around $\hat{z}$ from $\hat{y}$ to $\hat{x}$; this produces the grid in (c). The mapping is complete after these rotations, and we can then use the FDTD 2D TM ${ }^{\mathrm{z}}$ field components $\left\{E_{z}, H_{x}, H_{y}\right\}$ to represent the analytical 2D TEz field components $\left\{E_{y}, H_{z}, H_{x}\right\}$, respectively, with no modifications to the established FDTD formulation nor the analytical formulation.

\subsection{S-Parameters Extraction Methodology in FDTD}

S-parameters are often used to characterize a variety of electronic systems $[7,19,26]$. The methodology of finding S-parameters may be applied to 2D FDTD simulations quite expediently $[7,19]$.

We use the traditional definition of S-parameters [26], where the total voltage wave measured at each port in a system can be decomposed into incident and reflected waves, i.e., $\tilde{V}=\tilde{V}^{+}+\tilde{V}^{-}$, and those components can be used to evaluate S-parameters as in (6), where $m, n, k$ are port numbers, $\tilde{V}^{+}$is the incident wave, $\tilde{V}^{-}$is the reflected wave, and $\tilde{V}$ is the total wave.

$$
S_{m n}=\left.\frac{\tilde{V}_{m}^{-}}{\tilde{V}_{n}^{+}}\right|_{\tilde{V}_{k}^{+}=0 \forall k \neq n}
$$

In our FDTD simulations, we use a two-port system, so $\{m, n, k\} \in\{1,2\}$. This methodology may be further extended to systems with more than two ports. 


\subsubsection{Computing S-Parameters, Using FDTD}

We are able to record total, incident, and reflected fields in FDTD simulations, but these may not all be recorded simultaneously. Therefore, we utilize a four-step simulation setup for collecting S-parameters.

Other simulations for calculation of loss may be considered a subset of the four-step simulation process [19]. Each of the simulation steps are visualized in Figure 4, and explained below. The baseline setup and geometry is in Figure 1.
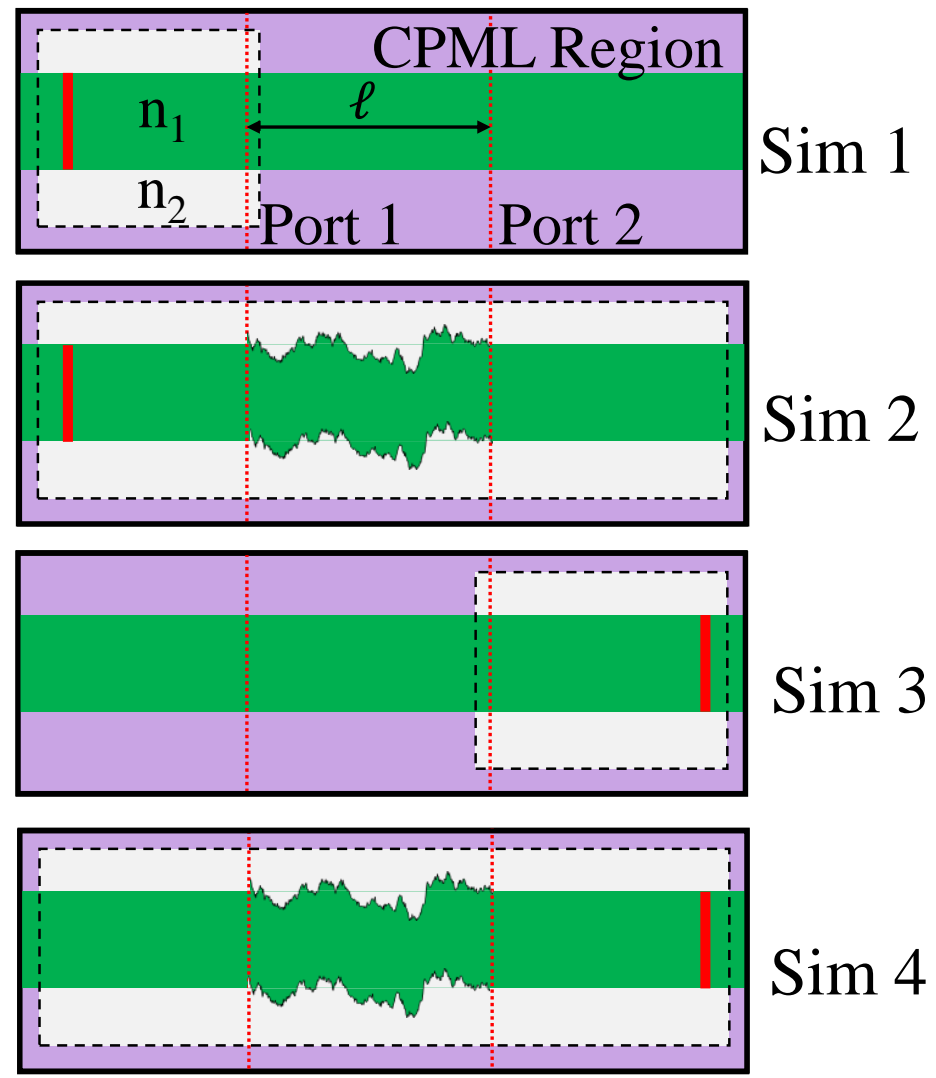

Figure 4. Four-step simulation setup for S-parameters extraction methodology.

Sim 1:The first simulation starts by placing the source condition at port 1 , so $\tilde{V}_{2}^{+}=0$ for this simulation. We simplify the geometry of the dielectric slab by excluding the random sidewall perturbations at this time. Additionally, we extend the right-side CPML boundary to 10 cells to the right of port 1 . This simulation results in $\tilde{V}_{1}^{+}$.

Sim 2: The second simulation starts with the same source condition as simulation 1. We then apply a valid discrete roughness profile to top and bottom boundaries between core and cladding. In our simulations, we chose the top and bottom profiles to be identical, but other choices are possible too. The CPML boundaries are evenly distributed around the computational domain. This simulation results in $\tilde{V}_{1}$ and $\tilde{V}_{2}^{-}$.

Sim 3: The third simulation is similar to the first simulation. We place the source condition at port 2, where $\tilde{V}_{1}^{+}=0$ in this simulation, and extend the left-side CPML boundary to 10 cells to the left of port 2. Simulation 3 results in $\tilde{V}_{2}^{+}$.

Sim 4: The fourth simulation finalizes the port field data collection. Similar to the third simulation, we place the source condition at port 2 , and similar to the second simulation we set the CPML boundaries at the baseline limits and apply the roughness profile in the same manner. This simulation results in $\tilde{V}_{1}^{-}$and $\tilde{V}_{2}$.

The fields recorded in the numerical experiments are limited to the four aforementioned steps, but there are still two field components missing which would fully describe the scattering matrix; those are $\tilde{V}_{1}^{-}$when $\tilde{V}_{2}^{+}=0$, and $\tilde{V}_{2}^{-}$when $\tilde{V}_{1}^{+}=0$. Here, we may 
use the decomposition relation to find the implicit reflected fields. Specifically, using $\tilde{V}_{1}^{+}$ from Simulation 1 and $\tilde{V}_{1}$ from Simulation 2, we may obtain $\tilde{V}_{1}^{-}=\tilde{V}_{1}-\tilde{V}_{1}^{+}$. Similarly, we may recover $\tilde{V}_{2}^{-}$from simulations 3 and 4.

With each of these values calculated from the numerical experiments, the S-parameters matrix may now be computed. In ideal waveguide with no sidewall perturbations, we would expect the S-parameters matrix (of the waveguide system) to be both symmetric (reciprocal) and unitary (lossless) [26]. For a waveguide with sidewall perturbations, we expect the S-parameters matrix (of the waveguide system) to be symmetric (reciprocal) but non-unitary (lossy) [26]. Higher port numbers may be simulated by following the same process of incident field simulation followed by total field simulation, for each port subsequently.

\subsubsection{Direct Method of Computing Scattering Loss, Using FDTD}

Using the second simulation, we may find the total voltage wave values at both ports 1 and 2 , but this time we label them $\tilde{V}(0)$ and $\tilde{V}(\ell)$, respectively. We assume the voltages to have the same form as the electric field but with the $\Phi(x)$ element replaced with $V_{0}$, i.e., $\tilde{V}(z)=V_{0} e^{-(\alpha+j \beta) z}$. In this form, it may be observed that as $z$ increases, we also expect the voltage to attenuate in amplitude and accumulate in phase.

Since we have measured the voltage at two port locations, we may determine the attenuation. To do this, we divide $\tilde{V}(\ell)$ by $\tilde{V}(0)$, resulting in (7).

$$
\frac{\tilde{V}(\ell)}{\tilde{V}(0)}=\frac{V_{0} e^{-(\alpha+\jmath \beta) \ell}}{V_{0}}=e^{-(\alpha+\jmath \beta) \ell}
$$

Using (7) we may isolate $\alpha$ by using the complex logarithm where $z \in \mathbb{C}, \log (z)$ is the complex-domain natural logarithm of $z, \ln (|z|)$ is the natural logarithm with base $e$, and $\arg ()$ is the true angle of $z$; i.e., the angle of $z$ which includes all full turns and may have a magnitude greater than $\pm \pi$ [27].

$$
\log (z)=\ln (|z|)+\jmath \arg (z),
$$

Applying (8) to (7) results in (9).

$$
-\alpha \ell-\jmath \beta \ell=\ln \left(\left|\frac{\tilde{V}(\ell)}{\tilde{V}(0)}\right|\right)+\jmath \arg \left(\frac{\tilde{V}(\ell)}{\tilde{V}(0)}\right)
$$

Equation (9) may be separated into real and imaginary components, resulting in the final expression in (10), for calculating power loss directly from FDTD experiments.

$$
\alpha=-\frac{1}{\ell} \ln \left(\left|\frac{\tilde{V}(\ell)}{\tilde{V}(0)}\right|\right) \quad(\mathrm{Np} / \mathrm{m})
$$

While (10) is possibly the most direct method for calculating power loss from FDTD simulation, there is an alternative definition which could accomplish that task through the use of S-parameters. We start by taking the argument of the natural logarithm in (10) and squaring it, but instead of 0 and $\ell$ being the reference points, the voltages are now in reference to ports 1 and 2 , leading to

$$
A=\left|\frac{\tilde{V}(\ell)}{\tilde{V}(0)}\right|^{2}=\left|\frac{\tilde{V}_{2}}{\tilde{V}_{1}}\right|^{2}=\left|\frac{\tilde{V}_{2}^{+}+\tilde{V}_{2}^{-}}{\tilde{V}_{1}^{+}+\tilde{V}_{1}^{-}}\right|^{2} .
$$

In (11), we may replace the magnitude-squared operation with the equivalent complex operation, resulting in

$$
A=\frac{\left(\tilde{V}_{2}^{+}+\tilde{V}_{2}^{-}\right)\left(\tilde{V}_{2}^{+}+\tilde{V}_{2}^{-}\right)^{*}}{\left(\tilde{V}_{1}^{+}+\tilde{V}_{1}^{-}\right)\left(\tilde{V}_{1}^{+}+\tilde{V}_{1}^{-}\right)^{*}},
$$


where * denotes the complex conjugate operator.

Simplifying (12), we may combine the incident and reflected voltage waves into compact S-parameters from. We may then reinsert $A$ into a natural logarithm and recover the expression for $\alpha$ as a function of S-parameters in (13) [19].

$$
\alpha=-\frac{1}{\ell} \ln \left(\left|\frac{\left(1+S_{11}\right)\left(S_{12}\right)}{S_{11}+S_{12} S_{21}}\right|\right) \quad(\mathrm{Np} / \mathrm{m}) .
$$

The form of $\alpha$ in (13) may also be utilized on systems with larger number of ports. In this case, port 1 is used as the reference port for loss, but in general the reference port may be any port in a multi-port system. In a large multi-port system, the loss equation using S-parameters may be computed for each reference port.

\subsection{Analytical Loss Function}

In the ensuing formulation, we assume simple media; i.e., linear, isotropic, and nondispersive. Following the recent work in [10], we propose (14) as the generic scattering loss function.

$$
\alpha=\frac{1}{N_{F}} \Phi^{2}(d) M_{W} S_{W} \quad(\mathrm{~Np} / \mathrm{m}) .
$$

Equation (14) is similar to the loss function used in previous works $[5,8,10]$, but we have made a modification by dividing $\alpha$ in those works by the normalization factor $N_{F}$.

Either part of the piece-wise function (2) may be evaluated at $x=d$ and result in (15).

$$
\Phi^{2}(d)=A_{e}^{2} \cos ^{2}(\kappa d),
$$

Note that $A_{e}$, derived in Equation (73) in [10], is dependent on the input power. This dependency is not desirable; thus, it is eliminated by introducing the factor, $N_{F}$, in (14), normalizing the amplitude of $\alpha$, as explained in Section 3.2.

The term $M_{W}$ is defined with (16).

$$
M_{W}=\left(n_{1}^{2}-n_{2}^{2}\right)^{2} \frac{k_{0}^{3}}{4 \pi n_{1}} .
$$

The term $S_{W}$ is defined with (17) and represents the contribution by the surface roughness described by a random distribution.

$$
S_{W}=\int_{0}^{\pi} \tilde{R}_{X X}\left(\beta-n_{2} k_{0} \cos (\theta)\right) d \theta,
$$

where $\tilde{R}_{X X}$ is the power spectral density of the surface roughness profile.

Surface roughness may be approximated as a stationary random process [10], therefore the power spectral density may be recovered through the ACF of the roughness profile, which may be assumed to have an exponential shape [4,5]. The exponential ACF is given by (18) [10]

$$
R_{X X}(\zeta)=\sigma^{2} e^{-\left|\frac{\zeta}{L_{c}}\right|},
$$

where $\sigma$ and $L_{c}$ are the standard deviation and correlation length of the profile, respectively, and $\zeta$ is the spatial shift variable.

There are two observations that could be made about (18) by setting $\zeta$ to specific values. First, $R_{X X}(\zeta=0)$ results in the variance of the profile. Second, $R_{X X}\left(\zeta=L_{c}\right)=\sigma^{2} e^{-1}$; this second result is used later for approximating the value of $L_{c}$ from surface profile data generated by the Pyspeckle software [22]. Some photo-lithographic processes for $\mathrm{Si} / \mathrm{SiO}_{2}$ may lead to profiles with $\sigma=9 \mathrm{~nm}$ and $L_{c}=50 \mathrm{~nm}$ [4].

We define the spatial Fourier transform (SFT) as

$$
\tilde{R}_{X X}(k)=\int_{-\infty}^{\infty} R_{X X}(\zeta) e^{-\jmath k \zeta} d \zeta,
$$


where the input function is translated from $\zeta$-space $(\mathrm{m})$ to $k$-space $(\mathrm{rad} / \mathrm{m}), k$ is the wave number, and the imaginary number $\jmath=\sqrt{-1}$.

Applying the SFT (19) to (18), yields

$$
\tilde{R}_{X X}(k)=\frac{2 L_{c} \sigma^{2}}{1+L_{c}^{2} k^{2}} .
$$

We may insert (20) into (17) and numerically evaluate the integral to obtain the contribution of the surface roughness profile to the loss $\alpha$.

\subsection{FDTD Model Validation}

We validate the FDTD model being used in these numerical experiments in three ways, described below. Unless stated otherwise, the data in this section is generated for a smooth waveguide with $\delta=300 \mathrm{~nm}, n_{1}=3.5, n_{2}=1.5$, and $f_{0}=194.8 \mathrm{THz}$. Validation must be done prior to performing numerical experiments, so we utilize the smooth waveguide and the below methods for model validation. Using known and expected attributes of the smooth waveguide, we can compare the results obtained from numerical experiments to provide confidence in the validity of our model before performing numerical experiments with waveguides that exhibit surface roughness.

\subsubsection{Wave Impedance}

One such method is through comparison with a known analytical solution to the smooth dielectric slab waveguide. We use the wave impedance of an outward traveling wave. This solution is well established and has been derived in several places [10,25]. We find the wave impedance by dividing $\mathrm{TE}^{\mathrm{Z}}$ mode E-field by the corresponding $\mathrm{H}$-field component along the length of the slab waveguide. Here, those fields are $E_{y}$ and $H_{z}$, respectively. In the smooth slab case, the real portion of the wave impedance should be very small. For the analytical solution, the division between the E-field and H-field gives

$$
Z_{w}=\jmath \frac{x}{|x|} \frac{\omega \mu}{\gamma}(\Omega),
$$

where $\mu$ is the magnetic permeability. Division of $x$ by its magnitude is used to set the appropriates sign for either above or below the slab.

The FDTD portion of this comparison may be conducted through the second simulation from Section 2.3.1 with the surface roughness omitted. Since the wave impedance is calculated for an outward traveling wave, we use the E-field and H-field data in the cladding region. We take all the steps necessary to compare frequency-domain voltages as described in Section 2.2, but we exclude the final integration such that we are left with field data for every point along the line at ports 1 and 2. Using the port 2 data allows for the wave to propagate over a long enough distance to be well-set into the lowest order mode. We take the measurements from two cells below the lowest core cell which leaves a single cell buffer between the core region and the cell used for this calculation. Finally, the imaginary component is compared to the analytical solution.

The wave impedance calculated from the FDTD model is shown in Figure 5a. We can see that the impedance found from numerical experiment matches with the expected analytical value throughout this range of frequency samples. At $f_{0}$ the difference between the FDTD and analytical values is approximately $1.5 \Omega$, which translates to an error of less than $2 \%$ near the frequency of interest. 


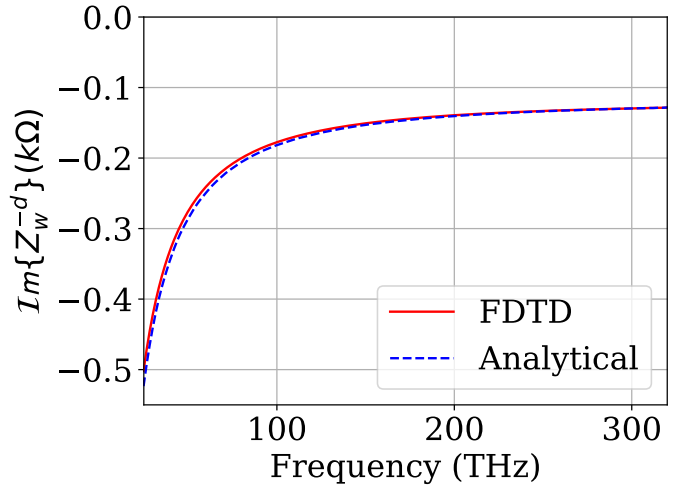

(a)
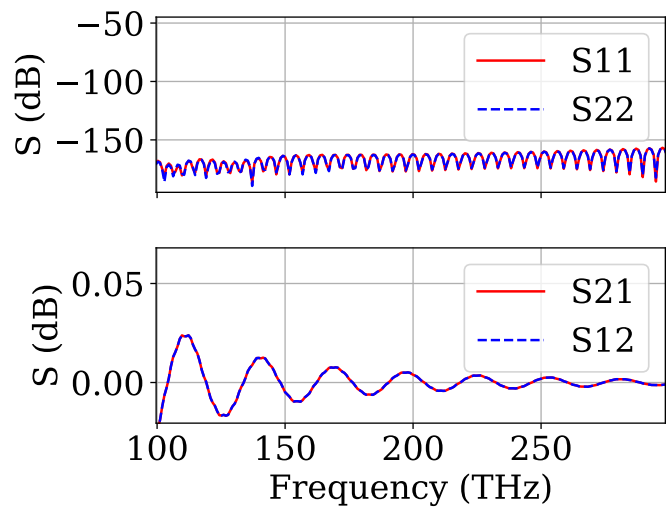

(c)

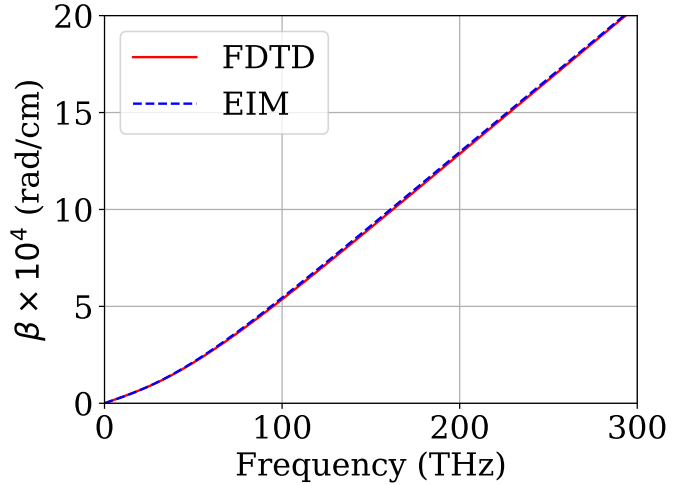

(b)

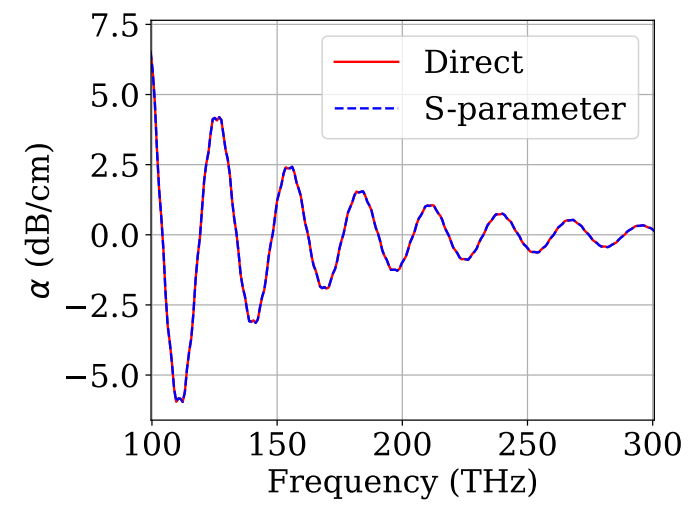

(d)

Figure 5. (a) Zeroth order $\mathrm{TE}^{\mathrm{z}}$ mode wave impedance for the smooth dielectric slab waveguide. (b) Propagation constant $\beta$ vs. frequency. (c) S-parameters for the smooth waveguide. (d) Propagation loss $\alpha(\mathrm{dB} / \mathrm{cm})$ vs. frequency, for a smooth waveguide with S-parameters method vs. direct method for calculating propagation loss.

\subsubsection{Propagation Constant}

In the next validation method, we compare the propagation constant $\beta$ obtained from FDTD against that obtained from the EIM in the frequency range of interest, at the same samples as the FDTD model output. We find $\beta$ from FDTD by evaluating the imaginary component of (9).

Since we are examining the phase angle of the voltage measurements, the division of $\tilde{V}(\ell)$ by $\tilde{V}(0)$ may be converted to a subtraction, resulting in (22).

$$
\beta=-\frac{1}{\ell}(\arg (\tilde{V}(\ell))-\arg (\tilde{V}(0)))
$$

In FDTD, we compute the angle of the complex voltages over the entire frequency range on the smooth dielectric slab waveguide and unwrap the final angle array into full angle form (rather than principal angle form).

In Figure 5b, the traces are nearly overlapped. The fundamental frequency is highlighted by the vertical dashed line, where the error between the EIM and the FDTD model is less than 1\%. This data further validates the FDTD model, and confirms the formulation leading to (22).

\subsubsection{Scattering Matrix}

In the last validation method, we utilize the properties of the S-parameters matrix described in Section 2.3.1. As stated, the matrix should be symmetric and unitary for the 
smooth waveguide. We extract the S-parameters from the FDTD model using the method presented in Section 2.3, and show these in Figure 5c.

Two observations are noteworthy in the frequency range of interest: (1) the crossterms $(\mathrm{S} 12, \mathrm{~S} 21)$ have a magnitude of near $0 \mathrm{~dB}$, indicating that there is almost complete transmission of power from one end of the waveguide to the other, and the self-terms (S11, S22) are correspondingly very small compared to the cross-terms, with a peak value of less than $-150 \mathrm{~dB}$; therefore, the matrix is nearly unitary as expected for a smooth lossless waveguide. (2) the S-parameter matrix is symmetrical, given the near perfect overlap of S11 with S22, and S12 with S21. These observations are noteworthy because they are the expected results for an ideal network, such as a smooth dielectric slab waveguide. Since the FDTD results align well with the expected behavior of a 2-port network, these results further validate the FDTD model.

We compare the S-parameters method of Section 2.3.1 and the direct method of Section 2.3.2 for calculating loss, as shown in Figure $5 \mathrm{~d}$. Here, we observe an oscillatory behavior similar to that in the cross-terms of Figure $5 \mathrm{c}$. The oscillations hover around $\alpha=0 \mathrm{~dB} / \mathrm{cm}$ and decay with increasing frequency, while the expected per-unit-length attenuation for an ideal smooth waveguide is $\alpha=0 \mathrm{~dB} / \mathrm{cm}$. Note that the loss from the S-parameters and from the direct method match very well, where the mean-squared error is on the order of $10^{-8}$.

\section{Results and Discussion}

Unless stated otherwise, the data in this section are generated for waveguides with $\delta=200 \mathrm{~nm}, n_{1}=3.5, n_{2}=1.5, f_{0}=194.8 \mathrm{THz}$, and $\Delta x=7.33 \mathrm{~nm}$.

In Figure 6, we show an example loss curve simulated in FDTD, to illustrate the need for filtering the FDTD output. As we can be seen in the figure, there is a nontrivial level of noise on the full range of $\alpha$.

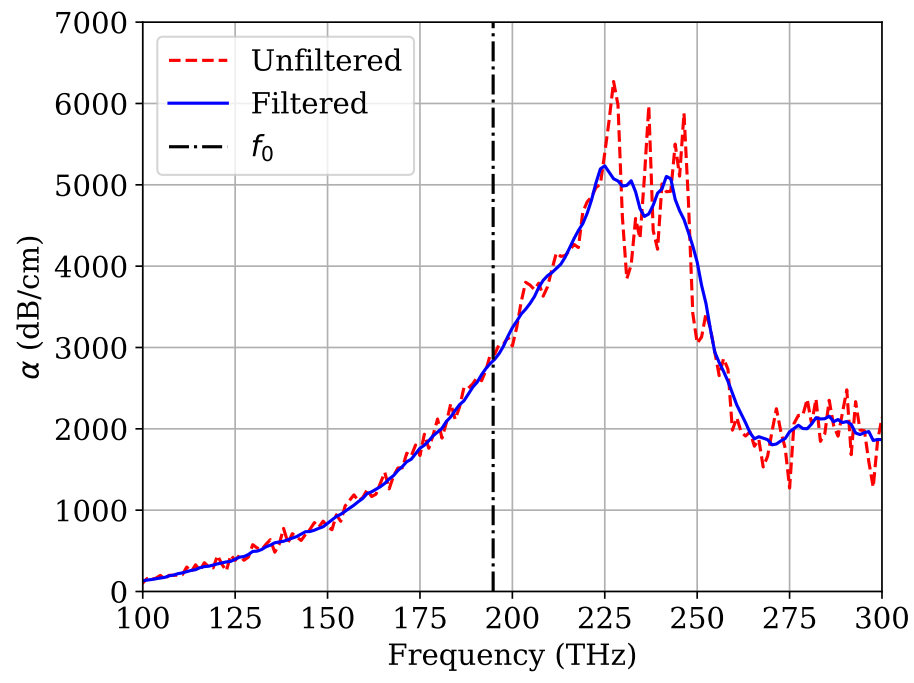

Figure 6. $\alpha$ vs. frequency, for a rough waveguide $\left(\sigma=15 \mathrm{~nm}, L_{c}=200 \mathrm{~nm}\right)$ with noisy FDTD data (red dashed line) compared to filtered FDTD data (blue solid line), where $f_{0}$ is the excitation source frequency.

Applying the filter described in Section 2.2.2 results in the Filtered trace which considerably reduces the noise in the FDTD data. This is best exemplified by the $\alpha$ values for frequencies above $225 \mathrm{THz}$, where the noise is reduced by an order of magnitude. The $\alpha$ values from FDTD are subjected to this filtering technique prior to calculation of the percentage error between the FDTD and the analytical solution (14).

Figure 7 uses the data from tables I and II in [7], respectively, to illustrate the distribution of percent error between FDTD and analytical calculations. Figure 7a,b were generated with the standard deviation $\sigma=9 \mathrm{~nm}$. The correlation length $L_{c}$ varies uniformly in the 
range $200 \mathrm{~nm}$ to $1000 \mathrm{~nm}$. The figures show the distribution of percentage errors for all correlation lengths with the same standard deviation, where a total of 924 roughness profiles were simulated using the FDTD model. We use these data to illustrate the effect of filtering on simulation results. In Figure $7 \mathrm{a}$ the mean error is $-5.12 \%$, whereas in Figure $7 \mathrm{~b}$ the error is reduced to $-4.12 \%$. Likewise, the standard deviation reduces from 21.96 to $19.87 \%$.

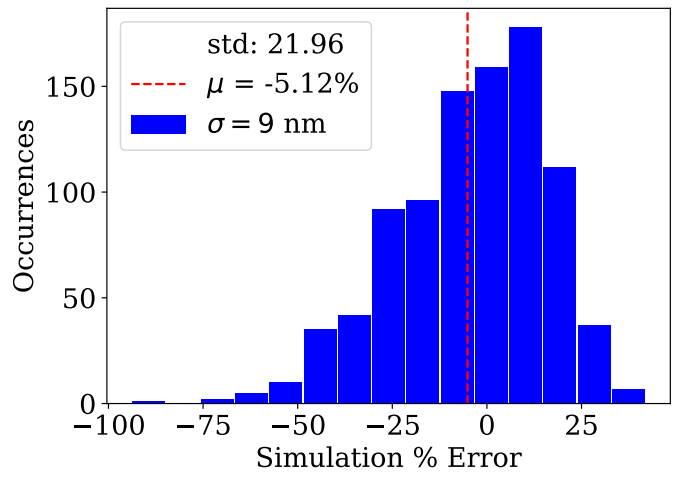

(a)

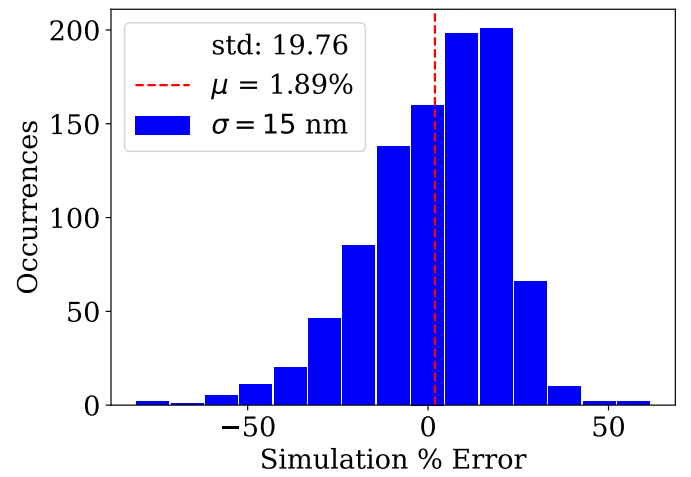

(c)

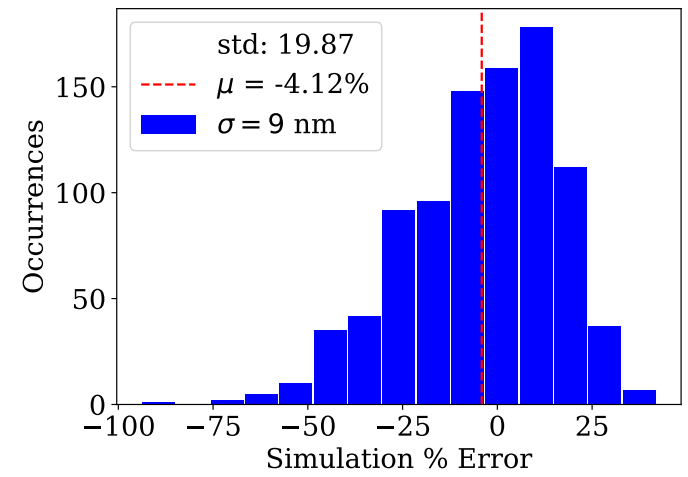

(b)

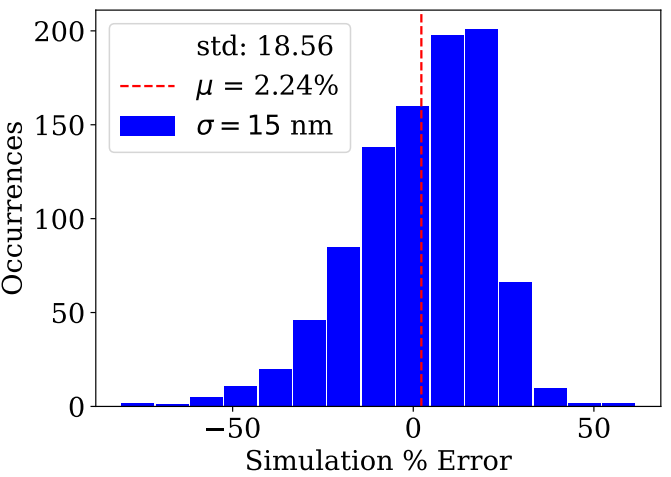

(d)

Figure 7. Percent error in propagation loss $\alpha(\mathrm{dB} / \mathrm{cm})$ between analytical (14) vs. FDTD solutions: (a) 924 roughness profiles at $\sigma=9 \mathrm{~nm}$, (b) same as (a) with data filtering, (c) 947 roughness profiles at $\sigma=15 \mathrm{~nm}$, and (d) same as (c) with data filtering.

A similar setup is used for Figure $7 \mathrm{c}, \mathrm{d}$, where $\sigma=15 \mathrm{~nm}$ and $L_{c}$ is uniform in the range $200 \mathrm{~nm}$ to $1000 \mathrm{~nm}$, with a total of 90 roughness profiles simulated with the FDTD model. In Figure $7 \mathrm{c}$ the mean error is $1.89 \%$, whereas in Figure $7 \mathrm{~d}$ the error is increased, to $2.24 \%$. Like in the $\sigma=9$ plots, the standard deviation reduces, this time from 19.76 to $18.56 \%$. From the numerical experiments conducted in FDTD on the relatively short-length slab waveguide, we have created the histogram of occurrences, which may be easily translated to a probability mass distribution.

Some potential sources of error in Figure 7 are listed below. (1) The parameters $\left\{\sigma^{\prime}, L_{c}^{\prime}, \mu^{\prime}\right\}$, rather than $\left\{\sigma, L_{c}, \mu\right\}$, are used in the analytical solution when calculating the percentage errors. (2) We use a relatively coarse spatial and temporal resolution in the FDTD model, while utilizing a finer resolution grid may decrease the percentage error range, it would increase computation time. (3) We use (14) as the analytical model, which is based on the formulation originally proposed in [5] that used various approximations and simplifications, such as a first-order Taylor series expansion to evaluate the E-field.

From each setup in Figure 7 the maximum difference between filtered and unfiltered data is standard deviation from Figure $7 \mathrm{a}$, b which reduces by $2.09 \%$, and each other relevant value changes by around 1\%, with the mean percentage error in the $\sigma=15 \mathrm{~nm}$ case actually increasing by $0.35 \%$. This shows that the filtering step does not significantly impact the percentage error of the FDTD results from the analytical solution. 
We define the improvement metric $F_{\mathrm{imp}}$ as

$$
F_{\text {imp }}\left(x_{\text {Filtered }}, x_{\text {Unfiltered }}\right)=\left(1-\frac{\left|x_{\text {Filtered }}\right|}{\left|x_{\text {Unfiltered }}\right|}\right) \times 100 \% \text {, }
$$

where $x_{\text {Filtered }}=\alpha_{\text {Filtered }}-\alpha_{\text {Analytical }}, x_{\text {Unfiltered }}=\alpha_{\text {Unfiltered }}-\alpha_{\text {Analytical }}, \alpha_{\text {Filtered }}$ and $\alpha_{\text {Unfiltered }}$ are defined in Section 2.2.2, and $\alpha_{\text {Analytical }}$ is defined by (14).

We use (23) to compare the effect of filtering on the FDTD data. Using this metric, we consider any value greater than $0 \%$ to be an improvement, and $100 \%$ would indicate the filter has maximally improved the unfiltered signal. We consider $\pm 5 \%$ to be negligible change, i.e., filtering had little effect on unfiltered signal. For $\sigma=9 \mathrm{~nm}$, filtering improves the error in 487 instances out of 924, and that filtering has negligible effect for 62 instances. For $\sigma=15 \mathrm{~nm}$ (23) results in improvement for 462 instances out of 947, and negligible effect for 107 instances.

It is worth noting that the above technique involves filtering $\alpha$ after $\mathrm{E}$ - and $\mathrm{H}$-fields have been computed in FDTD. The data show that filtering in this manner does not add significant value. Thus, we plan to investigate filtering techniques within FDTD during the computation of E- and H-fields, as was demonstrated in [28] for suppressing spurious noise waves due to sub-gridding.

\subsection{Comparing Our Results to Previous Publications}

We compare this work to previous theoretical and experimental works. Equation (14) is based on the formulation provided in [10], which in turn is based on both [5,8]. The primary difference between [10] and this work is that here we introduce the normalization factor $N_{F}$. In [10], the components for $\alpha$ are based on the more physically realistic form of E-field (2) where $A_{e}$ may be any real-valued scalar. The absence of the normalization factor (i.e., setting $N_{F}=1$ ) leaves a dependency on input power; however, the input power may be modified to fit the expected scattering loss value for any point, by setting (24) equal to (25) and solving for $\alpha$, which will always result in (29). In [5], the original formulation for finding scattering loss was proposed, where the mathematical normalization of (24) is used.

The follow-up work of [8] proposed a formulation for scattering loss calculations by using normalized waveguide parameters, but as was noted in [6], there appears to be an extra factor of 2 in the formulation used therein. We can see this numerically by comparing [8] with this work, also in Table 1, where the loss value is double what is calculated by (14). We further see that an input power of $P_{\mathrm{TE}}=4.3 \mathrm{~mW}$ in the formulation found in [10] is most similar to the normalized values found here, but other choices of input power (e.g., $P_{\mathrm{TE}}=1 \mathrm{~mW}$ or $P_{\mathrm{TE}}=1.45 \mathrm{~mW}$ ) could yield different values for loss as they may not eliminate dependence of $\alpha$ on input power.

The VCM was used in [6] to verify the scattering loss calculations. Their work was done on several 3D waveguides, and these provide a similar analogue to the 2D structure simulated in this work.

Looking at the experimental side, in [3] a physical 3D dielectric optical interconnect was tested for scattering loss. Their results show that the 2D planar model [8] is generally an overestimate of what can be expected from physical hardware, and the 3D simulations in [6] are generally an underestimate. Furthermore, in [3], unit variance is used, making it unique compared to other experimental data and included in Table 1. Other experiments conducted on physical hardware include those of $[1,4]$, where a scattering loss magnitude of $\approx 35 \mathrm{~dB} / \mathrm{cm}$ is reported. These experiments were conducted on 3D SOI optical interconnects consisting of $\mathrm{Si}$ core and $\mathrm{SiO}_{2}$ cladding extending $1 \mu \mathrm{m}$ in each direction around the core, making them amenable to comparison with the $2 \mathrm{D}$ planar approximation. The loss value in $[1,4]$ is approximately $36 \%$ of the loss value in (14), and approximately $72 \%$ of the loss value in [6]. 
Table 1. $\alpha$ values in $(\mathrm{dB} / \mathrm{cm})$.

\begin{tabular}{lcc}
\hline $\begin{array}{l}\text { Source } \\
\text { (a: hardware experimental) } \\
\text { (b: numerical/analytical) }\end{array}$ & $\begin{array}{c}d=210 \mathrm{~nm} \\
L_{c}=20 \mathrm{~nm}\end{array}$ & $\begin{array}{c}d=250 \mathrm{~nm} \\
L_{c}=50 \mathrm{~nm}\end{array}$ \\
\hline a [4] Lee & N/A & $\sigma^{2}=81 \mathrm{~nm}^{2}$ \\
\hline a [1] Jaberansary & $\mathrm{N} / \mathrm{A}$ & $\approx 34$ \\
\hline $\mathrm{a}[3]$ Horikawa & $\approx 0.5$ & $\approx 33$ \\
\hline $\mathrm{b}[6]$ Barwicz & $\mathrm{N} / \mathrm{A}$ & $\mathrm{N} / \mathrm{A}$ \\
\hline $\mathrm{b}[8]$ PL 94 & 1.87 & 48.6 \\
\hline $\mathrm{b}[10], P_{\mathrm{TE}}=1 \mathrm{~mW}$ & 0.22 & 193.7 \\
\hline $\mathrm{b}[10], P_{\mathrm{TE}}=1.45 \mathrm{~mW}$ & 0.32 & 22.1 \\
\hline $\mathrm{b}[10], P_{\mathrm{TE}}=4.3 \mathrm{~mW}$ & 0.94 & 32.1 \\
\hline This work $(14)$ & 0.94 & 95.2 \\
\hline
\end{tabular}

\subsection{Mode Normalization}

Although allowing $\alpha$ to have dependency on waveguide's structural attributes such as the surface roughness profile, material parameters, or even input wavelength is desirable, dependence on input power is not. In previous works [5,8], the E-field is mathematically normalized, such that

$$
\int_{-\infty}^{\infty} \Phi^{2}(x) d x=1
$$

While the above normalization removes the dependence of $\alpha$ on the input power, it also forces $\Phi(x)$ to satisfy a certain mathematical requirement; however, if we evaluate (24) by inserting the physical field expression (2) for the TE modes, we arrive at (25) [10].

$$
\int_{-\infty}^{\infty} \Phi^{2}(x) d x=A_{e}^{2}\left(d+\frac{1}{\gamma}\right) .
$$

Note that if (24) is assumed to be true, then the field amplitude $A_{e}$ would be forced to have the particular value $A_{e}^{2}=1 /\left(d+\frac{1}{\gamma}\right)$; however, this assumption may not hold true in the physical waveguide. To remedy the inconsistency, we start with [5]

$$
\alpha=\frac{P_{r a d} / 2 L}{P_{g}}=\frac{\frac{n_{2}}{2 \eta_{0}} \int_{0}^{\pi} \frac{\left\langle\left|E_{x}(r, \theta)\right|^{2}\right\rangle}{2 L} r d \theta}{\frac{n_{1}}{2 \eta_{0}} \int_{-\infty}^{+\infty} \Phi^{2}(x) d x},
$$

where the core and cladding refractive indices are tied to the guided power $P_{g}$ and the radiated power $P_{\text {rad }},\langle f\rangle$ designates the ensemble average of $f$ [10], and $\eta_{0} \approx 377 \Omega$ [5].

We use (15) from [5] to simplify the numerator in (26) and note that $2 \eta_{0}$ cancels at this point, resulting in (27).

$$
\alpha=\frac{n_{2}}{n_{1}} \frac{\Phi^{2}(d)\left(n_{2}^{2}-n_{1}^{2}\right)^{2} \frac{k_{0}^{3}}{4 \pi n_{2}} \int_{0}^{\pi} \tilde{R}_{X X}\left(\beta-n_{2} k_{0} \cos \theta\right) d \theta}{\int_{-\infty}^{+\infty} \Phi^{2}(x) d x} .
$$

We use the definition of $M_{W}$ and $S_{W}$ here to further simplify (27); this results in (28), where $n_{2}$ in the numerator cancels and the $n_{1}$ in the denominator is pulled into $M_{W}$.

$$
\alpha=\frac{\Phi^{2}(d) M_{W} S_{W}}{\int_{-\infty}^{+\infty} \Phi^{2}(x) d x}
$$

We may further simplify (28) for the zeroth-order mode by inserting the result from (25) and the physical field amplitude expression of $\Phi^{2}(d)$ in (15), followed by canceling the resulting $A_{e}^{2}$ term in the numerator and denominator. 


$$
\alpha=\frac{\cos ^{2}(\kappa d) M_{W} S_{W}}{d+1 / \gamma}
$$

Therefore, to remove dependence on input power uniformly for any initial input field amplitude, we propose to define $N_{F}$ in (14) as (30), where $N_{F}$ has the same dependence on input power as $\Phi^{2}(d)$.

$$
N_{F}=A_{e}^{2}\left(d+\frac{1}{\gamma}\right),
$$

Note that here, (29) is the simplest form for even TE modes, using the physical considerations based on actual field values in the waveguide. In [5], (24), is assumed to be true prior to calculating $\alpha$, forcing the denominator of (28) to be 1.0 and limiting $\Phi^{2}(d)$ to be single valued. The crucial difference in the proposed formulation here (based on inclusion of $N_{F}$ in $\alpha$ ) lies in the assumption that the E-field amplitude can be functionally any value. We believe that the proposed normalization based on $N_{F}$ offers a more physically consistent expression of $\alpha$, and works more intuitively for correlation of numerical (or physical) experiments in FDTD (or in the lab) where the initial field amplitudes may be specified with regard to considerations independent of scattering loss.

\section{Conclusions}

Based on physical waveguide parameters, an explicit normalization factor (30) for the scattering loss $\alpha$ (14) was proposed. The equation was then used to compare the results across several previous publications, including both numerical and physical experiments, showing that the analytical equation is generally an overestimation of actual propagation loss in a physical waveguide. We used the proposed analytical equation to confirm the presence of an extra factor of 2 in [8].

The proposed analytical formulation of scattering loss was verified, using an expedient FDTD scheme which included extraction of the attenuation coefficient and S-parameters. We validated the FDTD scheme by comparing numerical results against previously published analytical functions for the dielectric slab waveguide.

With the FDTD model verified, we demonstrated S-parameters extraction and attenuation coefficient calculation by applying the proposed methodology to a smooth dielectric slab waveguide. We then applied the methodology to compute the attenuation coefficient for a dielectric slab waveguide exhibiting random sidewall perturbations according to the exponential autocorrelation function. We proposed to use a filtering technique to reduce the signal-to-noise ratio of the final FDTD data in the frequency domain.

Along the way, we demonstrated the ability of the FDTD scheme to produce reasonably accurate results through tens of simulations for sidewall roughness profiles of varying correlation length at standard deviations of $\sigma=9 \mathrm{~nm}$ and $\sigma=15 \mathrm{~nm}$. The FDTD results showed that the mean error for simulation is quite small, with an overall average error of only $-4.12 \%$ and $2.24 \%$ for the attempted standard deviations, respectively.

The Python FDTD code (OIDT) [13] used to generate much of the data in this paper was released as open-source software (under the GNU GPL v3.0 license [15]) published on GitHub [14], featuring multi-core support (for CPU) to compute the 2D TEz fields of optical interconnects. Work is currently underway to develop efficient 2D transverse magnetic (TM), and full 3D, FDTD models for further characterization of stochastic scattering loss due to sidewall roughness in nano-scale optical interconnects, consisting of single-line and multiple tightly-coupled lines, operating at $100 \mathrm{~s}$ of THz.

Author Contributions: Conceptualization, A.Z.; Data curation, B.G.; Formal analysis, B.G. and A.Z.; Funding acquisition, A.Z.; Investigation, B.G. and A.Z.; Methodology, B.G. and A.Z.; Project administration, A.Z.; Resources, A.Z.; Software, B.G.; Supervision, A.Z.; Validation, B.G.; Visualization, B.G.; Writing-original draft, B.G.; Writing-review \& editing, A.Z. All authors have read and agreed to the published version of the manuscript. 
Funding: This work was funded, in part, by the NSF; Award \# 1816542 [13].

Institutional Review Board Statement: Not applicable.

Informed Consent Statement: Not applicable.

Data Availability Statement: Not applicable.

Conflicts of Interest: The authors declare no conflict of interest.

\section{References}

1. Jaberansary, E.; Masaud, T.M.B.; Milosevic, M.M.; Nedeljkovic, M.; Mashanovich, G.Z.; Chong, H.M.H. Scattering Loss Estimation Using 2-D Fourier Analysis and Modeling of Sidewall Roughness on Optical Waveguides. IEEE Photonics J. 2013, 5, 6601010. [CrossRef]

2. Poulton, C.G.; Koos, C.; Fujii, M.; Pfrang, A.; Schimmel, T.; Leuthold, J.; Freude, W. Radiation Modes and Roughness Loss in High Index-Contrast Waveguides. IEEE J. Sel. Top. Quantum Electron. 2006, 12, 1306-1321. [CrossRef]

3. Horikawa, T.; Shimura, D.; Mogami, T. Low-loss silicon wire waveguides for optical integrated circuits. MRS Commun. 2015, 6, 9-15. [CrossRef]

4. Lee, K.; Lim, D.; Luan, H.; Agarwal, A.; Foresi, J.; Kimerling, L. Effect of Size and Roughness on Light Transmission in a Si/SiO2 Waveguide Experiments and Model. Appl. Phys. Lett. 2000, 77, 1617-1619. [CrossRef]

5. Lacey, J.; Payne, F. Radiation Loss From Planar Waveguides with Random Wall Imperfections. IEE Proc. 1990, 137, 282-288. [CrossRef]

6. Barwicz, T.; Haus, H.A. Three-Dimensional Analysis of Scattering Losses Due to Sidewall Roughness in Microphotonic Waveguides. J. Light. Technol. 2005, 23, 2719-2732. [CrossRef]

7. Guiana, B.; Zadehgol, A. FDTD Simulation of Stochastic Scattering Loss Due to Surface Roughness in Optical Interconnects. In Proceedings of the 2022 United States National Committee of URSI National Radio Science Meeting (USNC-URSI NRSM), Boulder, CO, USA, 4-8 January 2022; pp. 1-2.

8. Payne, F.; Lacey, J. A Theoretical Analysis of Scattering Loss from Planar Optical Waveguides. Opt. Quantum Electron. 1994. 26, 977-986. [CrossRef]

9. Guiana, B.; Zadehgol, A. Stochastic FDTD Modeling of Propagation Loss due to Random Surface Roughness in Sidewalls of Optical Interconnects. In Proceedings of the United States National Committee of URSI National Radio Science Meeting (USNC-URSI NRSM), Virtual, 4-9 January 2021; pp. 266-267.

10. Zadehgol, A. Complex s-Plane Modeling and 2D Characterization of the Stochastic Scattering Loss in Symmetric Dielectric Slab Waveguides Exhibiting Ergodic Surface-Roughness with an Exponential Autocorrelation Function. IEEE Access 2021, 9, 92326-92344. [CrossRef]

11. Kuznetsov, M.; Haus, H.A. Radiation Loss in Dielectric Waveguide Structures by the Volume Current Method. IEEE J. Quantum Electron. 1983, QE-19, 1505-1514. [CrossRef]

12. Python Programming Language. Available online: https://www.python.org/ (accessed on 20 October 2021).

13. Zadehgol, A. SHF: SMALL: A Novel Algorithm for Automated Synthesis of Passive, Causal, and Stable Models for Optical Interconnects. National Science Foundation (NSF) Award \#1816542. Proposal submitted on 11/15/2017. Grant period: 10/1/2018-9/30/2021. Available online: https://nsf.gov/awardsearch/showAward?AWD_ID=1816542\&HistoricalAwards=false (accessed on 20 October 2021).

14. Guiana, B. Optical Interconnect Designer Tool (OIDT). Available online: https://github.com/bmguiana/OIDT (accessed on 23 November 2021).

15. GNU General Public License v3.0. Available online: https://www.gnu.org/licenses/gpl-3.0.en.html (accessed on 20 October 2021).

16. Yee, K.S. Numerical Solution of Initial Boundary Value Problems Involving Maxwells Equations in Isotropic Media. IEEE Trans. Antennas Propag. 1966, Ap14, 302.

17. Taflove, A.; Hagness, S.C. Computational Electrodynamics the Finite-Difference Time-Domain Method, 3rd ed.; Artech House Inc.: Norwood, MA, USA, 2005.

18. Roden, J.A.; Gedney, S.D. Convolution Pml (CPML) an Efficient Fdtd Implementation of the Cfs-Pml for Arbitrary Media. Microw. Opt. Technol. Lett. 2000, 27, 334-339. [CrossRef]

19. Guiana, B.; Zadehgol, A. S-Parameter Extraction Methodology in FDTD for Nano-Scale Optical Interconnects. In Proceedings of the 15th International Conference on Advanced Technologies, Systems and Services in Telecommunications, Nis, Serbia, 20-22 October 2021; pp. 1-4.

20. Zadehgol, A. Deterministic Reduced-Order Macromodels for Computing the Broadband Radiation-Field Pattern of Antenna Arrays in FDTD. IEEE Trans. Antennas Propag. 2016, 64, 2418-2430. [CrossRef]

21. Cooley, J.W.; Tukey, J.W. An Algorithm for the Machine Calculation of Complex Fourier Series. Math. Comput. 1965, 19, $297-301$. [CrossRef]

22. Pyspeckle. Available online: https:/ / pyspeckle2.readthedocs.io/en/latest/\# (accessed on 15 October 2021). 
23. Deserno, M. How to Generate Exponentially Correlated Gaussian Random Numbers; Department of Chemistry and Biochemistry UCLA: Los Angeles, CA, USA, 2002.

24. Smith, S.W. The Scientist and Engineer's Guide to Digital Signal Processing; California Technical Publishing: San Diego, CA, USA, 1997; Chapter 15, pp. 277-284.

25. Balanis, C.A. Advanced Engineering Electromagnetics, 2nd ed.; Wiley: Hoboken, NJ, USA, 2012; pp. 270-275. 428.

26. Pozar, D.M. Microwave Electronics, 4th ed.; Wiley: Hoboken, NJ, USA, 2012; pp. 178-188.

27. Brown, J.W.; Churchill, R.V. Complex Variables and Applications, 8th ed.; McGraw-Hill: New York, NY, USA, 2009; pp. 93-99.

28. Zadehgol, A.; Cangellaris, A.C. Isotropic Spatial Filters for Suppression of Spurious Noise Waves in Sub-Gridded FDTD Simulation. IEEE Trans. Antennas Propag. 2011, 59, 3272-3279. [CrossRef] 